\title{
New aspects for the physiotherapy of pushing behaviour, D. Broetz and H.-O. Karnath, Neurorehabilitation 20(2005), 133-138
}

\author{
Roberta B. Shepherd* and Janet Carr \\ School of Physiotherapy, Faculty of Health Sciences, The University of Sydney, Australia
}

We read this discussion paper with great interest. Understanding the mechanisms underlying motor behaviour after a brain lesion is critical to the development of more focussed and effective interventions. Dr Karnath has for some time been at the forefront of research on perceptual disorders underlying the clinical phenomenon called "contraversive pushing" that occurs in some individuals after stroke and the development of a standardised testing method that helps clarify diagnosis [9].

Some individuals early after stroke experience an altered perception of the body's orientation in space, and they express a feeling of certainty that their body is oriented upright when it is in fact tilted about $18^{\circ}$ toward the paretic side [9]. The pushing behaviour observed by physiotherapists appears secondary to this impairment. Interestingly, reports available suggest that the incidence of pushing behaviour appears to be variable, with incidence ranging as low as $10 \%$ [10]. This may be due in part to differences in testing procedure. However, our own observations and clinical sessions with physiotherapists in several countries, suggest to us another possible explanation, that the behavioural development may be a natural adaptive response to rehabilitation methods that have the potential to increase the

*Address for correspondence: Honorary Professor Roberta Shepherd, 22 Albion Street, Waverley, NSW 2024, Australia. E-mail: R.Shepherd@fhs.usyd.edu.au. fear of falling and provoke a defensive pushing. For example, attempts by the therapist to move the patient into an upright position passively are resisted strongly as externally imposed movement to the non-paretic side is perceived as a major threat to balance and engenders considerable fear.

It is the patient's resistance to being moved passively by the therapist that some therapists have termed 'pushing' [5]. The problem to be remedied therefore is the individual's misperception of what constitutes an upright position. In an earlier paper Karnath and colleagues [8] suggested an intervention plan directed specifically at this problem, based on the findings of their research into underlying mechanisms.

We have a few suggestions to add this discussion. We have noted previously that when an individual demonstrates difficulty sitting erect, falls to the paretic side, and is fearful of falling, pushing behaviour is less evident under certain training conditions. These include early and active training that is task-oriented and takes into account both the impairment in perception of body alignment in space and the fear of falling [1,2]. We proposed that initially the patient could practise reaching for an object toward the paretic side which provides a means of actively controlling movement of the body mass to the side to which the person leans or falls, but without evoking fear. Active movement to the paretic side and moving back up into sitting are practised repetitively. Throughout training the person 
is encouraged to understand the mismatch between the true visual vertical and their own incorrect sense of the body's relation to gravity.

The patient learns to move the body mass over the base of support in the context of functional actions; that is, attention is paid to the object to be reached and where it is in space rather than to the body movement itself. If the arm is paralysed the therapist supports it but movement of the body mass laterally and back to sitting is performed actively by the patient. Emphasis is placed on the patient using the foot of the paretic leg for support, balance and to propel the body mass back into sitting. As proposed by Karnath and colleagues [8], the patient is encouraged to attend to critical visual cues such as vertical objects in the immediate environment (eg bed leg, door frame). The attentional strategy may help the person 'intellectualise' the situation and to concentrate on over-riding the mismatch between the subjective (but incorrect) perception of position in space and visual reality. This emphasis on active training fits within our current understanding of the need for patients after stroke to undergo active, task-oriented training and exercise, and is based on an understanding of the impairments and of biomechanical and motor control mechanisms.

The perceived likelihood of falling and therefore fear are increased if the patient is sitting on an elevated bed and the feet are not in contact with a stable support surface. Sitting with feet unsupported makes it more difficult to balance than with feet on the floor. Chari and Kirby's findings illustrate this, showing that young able-bodied subjects were significantly handicapped in their ability to reach in sitting when the feet were unsupported [3]. Biomechanical data have made it clear that the lower limbs play a critical role in supporting, shifting and balancing the body mass in sitting [4,6,7]. For example, shifting body weight laterally is brought about in part by pressure downward through the feet [7].

Sitting with feet unsupported therefore makes it difficult for the person to regain a sense of balance and move to an upright position as it increases instability and prevents the critical contribution of vertical force through the feet. Figure 3 in the Discussion Paper illustrates this point. Contrary to the caption, the person in the photograph has not shifted her body mass to the right. She is reaching toward the right but her body weight remains supported on the thigh and arm of the paretic side.

There is a related issue. Rather than focussing on teaching 'transfers', training of standing up and sitting down should also be started very early since inde- pendent sit-to-stand is essential for independence and loading the paretic leg in standing and walking. Practice of standing up and sitting down is made easier by raising the seat height. This reduces the muscle force required [11] and the horizontal distance to be moved. Similarly, treadmill training with partial body weight support enables practice of weight-bearing through the paretic limb.

In conclusion, drawing the patient's attention to vertically-oriented visual cues, as described in the Discussion paper, is a rational strategy for helping the person understand the mismatch between their 'feeling' of where they are in space and reality. Concentrating on the non-paretic limbs as substitutes is probably not, and may reinforce learned non-use of the paretic limbs. Aligning and balancing the body mass in sitting requires controlled and activity-specific muscle activity of both limbs and trunk with emphasis on loading the lower limbs. The patient's impairments include muscle weakness and paralysis of the lower limb, predominantly those muscles that cross hips, knees and ankles. Our current scientific understanding supports the use in clinical practice of exercise and training directed toward motor and perceptual impairments with a focus on training motor control in functional actions, strength training, and training to pay attention to appropriate visual and environmental cues.

If pushing behaviour develops as a secondary phenomenon, there is a strong possibility that it arises and is augmented by the patient's early experiences of passive corrections and external support by the therapist, with no training to re-establish a sense of balance and the ability to orient appropriately to gravity. Procedures that make the patient fearful of moving and of being forced off balance should be discontinued and replaced by methods more congruent with modern scientific understanding.

\section{References}

[1] J.H. Carr and R.B. Shepherd, Neurological Rehabilitation Optimising Motor Performance, Butterworth Heinemann, Oxford, 1998.

[2] J.H. Carr and R.B. Shepherd, Stroke Rehabilitation Guidelines for Exercise and Training to Optimise Motor Skill, Butterworth Heinemann, Oxford, 2003.

[3] V. Chari and R.L. Kirby, Lower-limb influence on sitting balance wile reaching forward, Arch Phys Med Rehabil 67 (1986), 730-733.

[4] J. Crosbie, R.B. Shepherd and T.J. Squires, Postural and voluntary movement during reaching in sitting: the role of the lower limbs, J Hum Mov Stud 28 (1995), 103-126. 
[5] P.M. Davies, Steps to Follow, A Guide to the Treatment of Adult Hemiplegia, Springer, New York, 1985, 2000.

[6] C. Dean, R. Shepherd and R. Adams, Sitting balance1: trunkarm and the contribution of the lower limbs during self-paced reaching in sitting, Gait Posture 10 (1999), 135-144.

[7] C. Dean, R.B. Shepherd and R.D. Adams, Sitting balance11: reach direction and thigh support affect the contribution of the lower limbs when reaching beyond arm's length in sitting, Gait Posture 10 (1999), 147-153.

[8] H.-O. Karnath and D. Broetz, Understanding and treating Pusher Syndrome, Physical Therapy 83 (2003), 1119-1125.
[9] H.-O. Karnath, S. Ferber and J. Dichgans, The origin of contraversive pushing: Evidence for a second graviceptive system in humans, Neurology 55 (2000), 1298-1304.

[10] P.M. Pedersen, A. Wandel, H.S. Jorgenson, H. Nakayama, H.O. Raaschou and T.S. Olsen, Ipsilateral pushing in stroke: incidence, relation to neuropsychological symptoms, and impact on rehabilitation. The Copenhagen Stroke Study, Arch Phys Med Rehabil 77 (1996), 25-28.

[11] M.V. Rodosky, T.P. Andriacchi and G.B.J. Andersson, The influence of chair height on lower limb mechanics during rising, J Orthop Res 7 (1989), 266-271. 